\title{
A SURVEY ON PERFORMANCE OF FREE SPACE OPTICAL COMMUNICATION LINK
}

\author{
Er. Sushila Thakur, Er. Rajeev Thakur \\ M.Tech student (ECE), Eternal University, Baru Sahib \\ Assistant Professor (ECE), Eternal University, Baru Sahib
}

\begin{abstract}
The modern era of communication needs a system with high bandwidth and high speed datarates. To fulfill rapid demands in today's technology era, Free space optical (FSO) communication system has become very attractive technology, because they provides high bandwidth, license free spectrum using infrared beam, and are a reliable means of communications. The first part of the paper introduces the FSO system its block diagram and also the reason behind its usage. The second part informs about the factors which impairs the performance of the system. The third part will define the factors which enhance the performance of FSO system. The fourth part here will discuss which type of frequency is better for FSO system. The last part will describe about the different modulation techniques and which modulation is good for FSO system.
\end{abstract}

\section{Indexing terms/Keywords}

Free Space Optics (FSO); Radio Frequency (RF); On-Off-Keying (OOK); Non-Return-to-Zero (NRZ); Return-to-Zero (RZ); Index-of-Refractive Turbulence (IRT); Avalanche Photodiode (APD); Vertical-cavity surface emitting laser (VCSEL); Field Of Vision (FOV); Inter Symbol Interference (ISI); Amplitude Shift keying (ASK); Differential Phase Shift keying (DPSK)

\section{Council for Innovative Research}

Peer Review Research Publishing System

\section{Journal: INTERNATIONAL JOURNAL OF COMPUTERS \& TECHNOLOGY}

Vol. 14 , No. 12

www.ijctonline.com, editorijctonline@gmail.com 


\section{INTRODUCTION}

Over the last two decades free-space opticalcommunication (FSO) has become more and more interesting as an adjunct or alternative to radio frequency (RF)communication.FSO has become a major topic in world of wireless and optical communications. It provides a high data rates up to several Gbps, immune to radio frequency interferences, requires no licensing, gives a highly secured communication link due to the usage of a very narrow beam angle and offers an inexpensive, fast and easy deployment when compared to the fiber optic installation [1].

\subsection{Free Space Optical Communication System}

Free Space Optics (FSO) is an optical communication technology that uses light propagating in free space to transmit data between two points. This technology is useful where a fiber optic cable is impractical. It is similar to fiber optic communications in that data is transmitted by modulated laser light. Instead of containing the pulses of light with in a glass fiber, these are transmitted in a narrow beam through the atmosphere. Light travels through air faster than glass[2].FSO systems are sensitive to bad weather conditions such as fog, haze, dust, rain and turbulence. All of these conditions act to attenuate light and could block the light path in the atmosphere[30].

If FSO systems are to be used in telecommunication applications, it needs to meet much higher availability requirements. Carrier-class availability is generally considered to be $99.999 \%$. For enterprise applications, link availability requirements are generally greater than $99 \%$. The enterprise market is where the majority of FSO systems have been deployed [3].

Transmitter

Atmospheric channel

Receiver

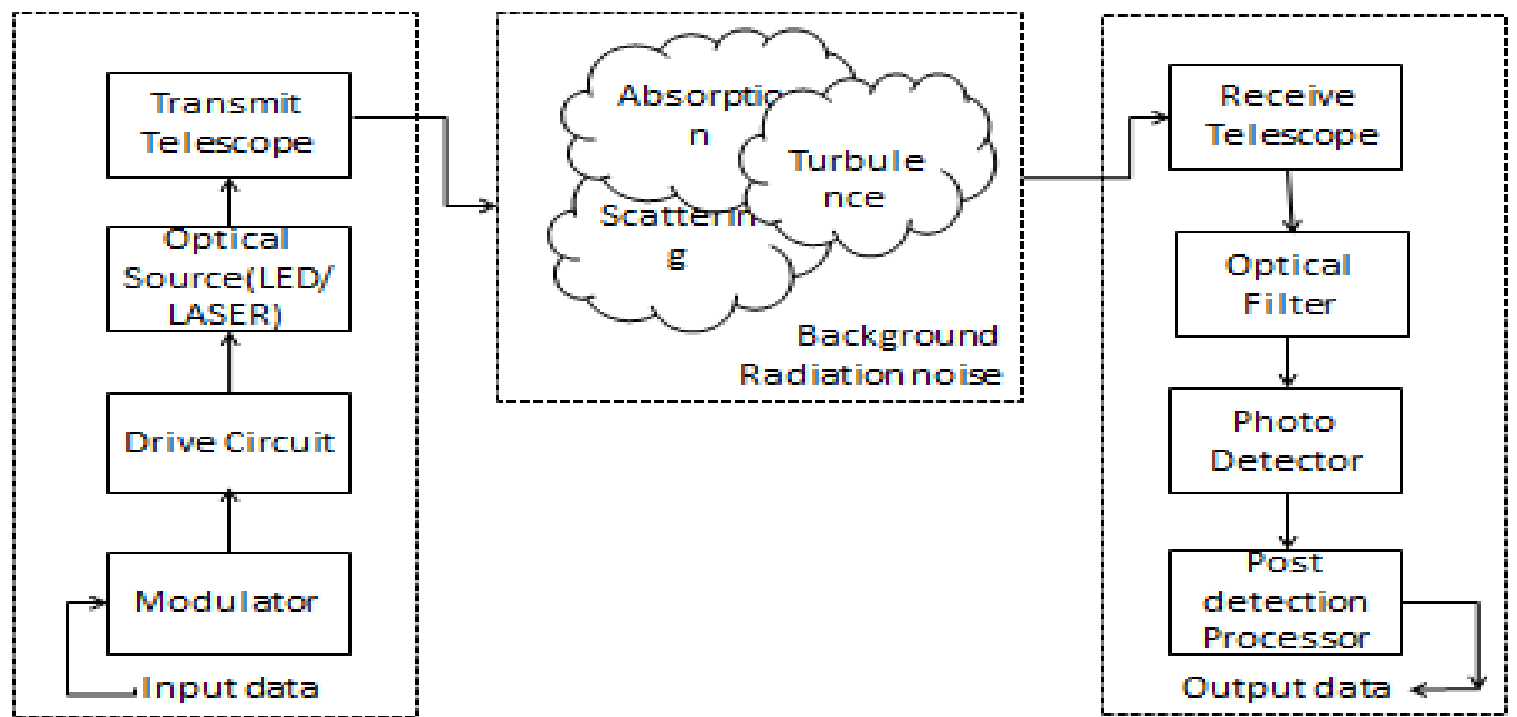

Figure1: Block diagram of FSO link

A free space optics communication system consists of three main systems namely the transmitter, the communication channel and the receiver. The transmitter is composed of the modulator, driver circuit, optical source and the transmitter telescope. The modulator is responsible for modulating the source message into the optical carrier. OOK is an intensity modulation scheme where the light source (carrier) is turned on to transmit a logic "one" and turned off to transmit a "zero". In its simplest form this modulation scheme is called NRZ (non-return-to-zero)-OOK. Besides NRZ other codes also exist. The most common one besides NRZ is RZ (return to-zero) coding. The advantages of RZ compared to NRZ are its higher sensitivity and the fact that the clock frequency lies within the modulation spectrum [20].

The optical source used in FSO systems can either be LED or laser. LEDs are often considered in transmitter design for their low cost and long lifetime, but they suffer from lower power. As a result of the lower power, LEDs are only suited for communications over short distances. A popular alternative to LEDs is the laser diode. The laser diode has the benefits of being small, having a characteristically high power, and is cost efficient [23].The transmitter telescope collects, collimates and directs the optical radiation from the optical source to the receiver at the other end of the channel [25].

For FSO the transmission channel is the atmosphere. The propagation of optical signals through free space is affected by three main processes namely absorption, scattering and Index-of-Refractive Turbulence (IRT).

The optical receiver is used to recover the transmitted information from the incident optical field. The optical receiver essentially comprises the receiver telescope, optical filter, photo detector and the post detection processor.The incoming optical radiation is collected by the receiver telescope and focused into the photo detector. A large receiver telescope 
aperture is desirable as it collects multiple uncorrelated radiations and focuses their average on photo detector. This is refer to as aperture averaging but a wide aperture also means more background radiation/ noise. Optical filter is used to reduce the background radiations. The photo detector collects the incident optical radiation from the receiver telescope and converts it back into electrical signal. P-I-N diode or Avalanche Photodiode (APD) is employed to do the optical and electrical channelconversion. The post detection processor or decision circuitry does the amplification, filtering and signal processing necessary to guarantee a highly reliable data recovery [25].

\subsection{Why FSO?}

Wireless communication is among technology's biggest contributions to mankind. Wireless networks have been dominated by IEEE 802.11 standards [4]. Wireless communication involves the transmission of information over a distance without help of wires, cables or any other forms of electrical conductors. The transmitted distance can be anywhere between a few meters (for example, a television's remote control) and thousands of kilometers (for example, radio communication) [22].Wireless networks are cheaper to install and maintain.

In wireless communications a line-of- sight technology is establishes a communication link between transmitters andreceivers by transmitting a laser beam through the atmosphere. The astonishing growth of internet traffic over the last decade by the general public has caused the phenomenon that is referred to as the "last mile bottleneck." The "last mile bottleneck" is the shortage in bandwidth that is a result of the connectivity technologies in metropolitan areas being unable to keep up with the increasing needs [10].

In order to meet the bandwidth demands, a number of communication links are under investigation including fiber optics, radio-frequency (RF) communications, copper-based technology, and free-space optics (FSO). The problems with the first three technologies stem from the high costs of installation, the slow time-to-market, the heavy investments in spectrum licenses, and the poor scalabilities of such technologies. In comparison, FSO links offer an optimal solution in regards to the given issues [23].

\subsection{LinkMarginEquation}

Link margin also called as fade margin is the percentage of time that the link is operating satisfactorily and when the link margin is not exceeded is known as link availability. The performance of a FSO link is calculated by using a link margin equation [26] given as follows

Link margin $(\mathrm{dB})=P_{e}+\left|S_{r}\right|-A t t_{\text {geo }}(\mathrm{dB})-A t t_{\text {atm }}(\mathrm{dB})-A t t_{\text {rain }}(\mathrm{dB})-A t t_{\text {scin }}(\mathrm{dB})$

Att $_{\text {snow }}$......

where,

$P_{e}$ is total power of emitter $(\mathrm{dBm})$

$\left|S_{r}\right|$ is sensitivity of receiver $(\mathrm{dBm})$

$A t t_{\text {geo }}$ is geometrical attenuation due to transmit beam spreading with increasing range $(\mathrm{dB})$

$A t t_{a t m}$ is atmospheric attenuation (dB)

$A t t_{\text {rain }}$ is rain attenuation $(\mathrm{dB})$

Att $_{\text {scin }}$ is scintillation attenuation $(\mathrm{dB})$

Att $_{\text {snow }}$ is snow attenuation $(\mathrm{dB})$

\subsection{Network Protocol- Transparent or Managed}

For carriers today the issue of interoperability of systems within their multi-faceted networks made up of both legacy and next generation networks is crucial. Most Free Space Optics (FSO) systems currently available are physical layer devices that act the same way as fiber optic cables and receivers and are therefore able to work with all protocols while not being limited to any of them. There are systems on the market that incorporate ATM into the device but most designers of Free Space Optics (FSO) systems have opted for a protocol 'transparent' approach for both deployment flexibility and costreduction. Should a carrier wish to add such switching functionality to networks incorporating physical layerproducts there are many switches available on the market, all of which will interoperate with a physical layer device [8].

\section{FACTORS IMPAIRING THE PERFORMANCE OF FSO SYSTEM}

The degradation of signal is mainly caused due to effect of climatology andalignment issues. Factors such as atmospheric attenuation (fog, rain and snow), Scattering,Scintillation, window attenuation, alignment or building motion causes to impair and reduce visibility affecting its performance.

\subsection{Atmospheric Attenuation}

Atmospheric attenuation is defined as the process whereby some or all of the electromagnetic wave energy is lost when traversing the atmosphere. Thus, atmosphere causes signal degradation and attenuation in a FSO system link. 


\subsubsection{Fog Attenuations}

Fog can be described as a cloud of small particles of water, smoke, ice or combination of these near the earth surface thereby scattering the incident light and hence reducing the visibility. Attenuation of the optical signal at a distance $\mathrm{R}$, due to fog [6] is determined by the Beer-Lambert law given as

$A t t_{f o g}=e^{\alpha(f o g)} \mathrm{R}$

Here $\alpha$ (fog) is the attenuation coefficient, given by

$\alpha(f \circ g)=\frac{10 \log V}{V(k m)}\left(\frac{\lambda}{\lambda_{0}}\right)^{-q}$

Here $V(\mathrm{~km})$ stands for visibility, $\lambda$ stands for wavelength and $\lambda_{0}$ as visibility wavelength reference $(550 \mathrm{~nm})$, and $\mathrm{q}$ is the size distribution coefficient of scattering. $q$ can be obtain by utilizing the Kim or Kruse models.

For Kruse model $q$ is given as,

$q= \begin{cases}1.6 & \text { if } \mathrm{V}>50 \mathrm{~km} ; \\ 1.3 & \text { if } 6 \mathrm{~km}<\mathrm{V}<50 \mathrm{~km} ; \\ 0.585 \mathrm{~V}^{\frac{1}{3}} & \text { if } \mathrm{V}<6 \mathrm{~km} .\end{cases}$

For Kim model $q$ is given as,

$q= \begin{cases}1.6 & \text { if } \mathrm{V}>50 \mathrm{~km} ; \\ 1.3 & \text { if } 6 \mathrm{~km}<\mathrm{V}<50 \mathrm{~km} ; \\ 0.16 \mathrm{~V}+0.34 & \text { if } 1 \mathrm{~km}<\mathrm{V}<6 \mathrm{~km} ; \\ \mathrm{V}-0.5 & \text { if } 0.5 \mathrm{~km}<\mathrm{V}<1 \mathrm{~km} ; \\ 0 & \text { if } \mathrm{V}<0.5 \mathrm{~km}\end{cases}$

\subsection{Scattering}

Scattering refers to the pinball machine nature of light trying to pass through the atmosphere. Light scattering can drastically impact the performance of FSO systems. Scattering is not related to a loss of energy due to a light absorption process. Rather, it can be understood as a redirection or redistribution of light that can lead to a significant reduction of received light intensity at the receiver location [5]. The scattering coefficients from the concentration of the particles and the effective cross section are given as[31]:

$\beta_{\text {scat }}=\alpha_{\text {scat }} N_{\text {scat }}[1 / \mathrm{km}]$........

where,

$\beta_{\text {scat }}$ is either $\beta_{m}$ or $\beta_{a}$

$\alpha_{\text {scat }}$ is the cross-section parameter, $N_{\text {scat }}$ is particle concentration $\left[1 / \mathrm{km}^{3}\right]$.s

\subsubsection{Rayleigh (molecular) Scattering}

A radiation incident on the bound electrons of an atom or molecule induces a charge imbalance or dipole that oscillates at the frequency of the incident radiation [5]. The Rayleigh scattering coefficient is given as follows [31]:

$\beta_{m}=\alpha_{m} N_{m}[1 / \mathrm{km}]$

Where, $\alpha_{a}$ is the Mie scattering cross-section, $N_{a}$ is number density of air particles $\left[1 / \mathrm{km}^{3}\right]$.

\subsubsection{Mie (aerosols) Scattering}

The Mie scattering regime occurs for particles about the size of the wavelength. Therefore, in the near infrared wavelength range, fog, haze, and pollution (aerosols) particles are the major contributors to the Mie scattering process [5].The attenuation due to Mie scattering can reach values of hundreds of $\mathrm{dB} / \mathrm{km}$ [32], [33]. The Mie scattering coefficient expressed as follows [31],

$\beta_{a}=\alpha_{a} N_{a}[1 / \mathrm{km}]$

Where, $\alpha_{a}$ is the Mie scattering cross-section $\left[\mathrm{km}^{2}\right], N_{a}$ is number density of air particles $\left[1 / \mathrm{km}^{3}\right]$. 


\subsection{Absorption}

Atoms and molecules are characterized by their index of refraction [5].The absorption coefficient depends on the type of gas molecules, and on their concentration. The absorption coefficient is expressed as follows [2].

$\beta_{a b s}=\alpha_{a b s} N_{a b s}\left[\frac{1}{k m}\right]$.

$\alpha_{a b s}$ is the effective cross section of the absorption particle $\left[\mathrm{km}^{2}\right], N_{a b s}$ is the concentration of the absorption particles $\left[1 / \mathrm{km}^{3}\right]$.

\subsection{Scintillation Loss}

Atmospheric turbulence results due to change in temperature and pressure which in turn causes scintillations [24]. When the beam is scintillated, photons of light are temporally steered by pockets of air in random direction. The received signal level at the detector fluctuates due to thermally induced changes in the index of refraction of the air along the transmit path. For the case of free-spaceoptics, this implies horizontal path propagation and therefore stronger scintillation [7]. To overcome the scintillation effects automatic gain control mechanism is used and also clock recovery phase lock loop time constant eliminates the effects of scintillation and jitter transference [8].Also the effect of integrating over the intensity reduces effect of scintillation. This reduction is called aperture averaging [24].

\subsection{Physical Obstruction}

Flying birds or construction cranes can temporarily block a single-beam FSO system, but this tends to cause only short interruptions, and transmissions are easily and automatically resumed. Optical wireless products use multi-beam systems (spatial diversity) to address temporary obstructions, as well as other atmospheric conditions, to provide for greater availability [9].

\subsection{Building Sway}

It is not intuitive to think that stationary buildings move, but they do. These buildings move due to sway that is caused by high winds, the thermal response of their materials and seismic activity. The higher you go in building more sway is present. The problem can be dealt with in two complementary ways: through beam divergence and active tracking [10].

\subsection{Alignment}

One of the key challenges with FSO systems is maintaining transceiver alignment. FSO transceiver transmit highly directional and narrow beams of light that must impinge upon the receive aperture of the transceiver at the opposite end of the link. For a FSO link to function, it is very important that both the transmitted beam of light and the receive field of vision (FOV) cone encompass the transceiver at the opposite end of the link [11]. Misalignment effects can reduce the capacity and increase the outage probability of the FSO link and therefore the signal needs to be tracked to maintain a perfect alignment.

\subsection{Solar Interference}

Solar interference in FSO systems operating at $1550 \mathrm{~nm}$ can be combatted in two ways. Firstly by using a long pass optical filter window to block all optical wavelengths below $850 \mathrm{~nm}$ from entering the system and secondly by using optical narrow band filter preceding the receiver detector to filter all but the wavelength actually used for communication. To handle the off axis solar energy two spatial filters are used. These sub systems allow FSO links to operate unaffected by solar interference that is more than 1.5 degrees off axis [8].

\subsection{Window Attenuation}

The FSO gives an advantage that it provides service through the windows for those customers who do not have the access to the terrace of their building or the apartment. But along with this advantage there is a limitation also, the glass of windows add some amount of the attenuation to the signal. Uncoated glass attenuates $4 \%$ per surface due to reflection. Tinted or insulated windows can have much greater signal attenuation [11].

\section{PERFORMANCE ENHANCEMENT OF FSO}

There are number of ways for mitigating the turbulence effects. These can be mitigated by using diversity techniques, multiple transmitters and adaptive optics [12].

\subsection{Diversity Scheme}

It refers to a method for improving the reliability of a message signal by using two or more communication channels with different characteristics. The diversity techniques are classified under three domains namely, temporal diversity, frequency diversity and spatial diversity.

\subsubsection{Spatial Diversity}

Among all the diversity techniques, spatial diversity with multiple transmitting and receiving antennas are most popular due to its efficiency in terms of system resource usage (no extra power and bandwidth utilization necessary).Spatial diversity 
provides a more robust and reliable communication and by introducing additional degrees of freedom to the system, it provides a higher system capacity without requiring any additional power or bandwidth [13].

\subsubsection{Temporal Diversity}

Multiple versions of the same signal are transmitted at different time instants. Alternatively, a redundant forward error correction codeis added and the message is spread in time by means of bit-interleaving before it is transmitted. Thus, error bursts are avoided, which simplifies the error correction [13].

\subsubsection{Polarization Diversity}

Multiple versions of a signal are transmitted and received via antennas with different polarization [13]. Polarization Diversity: Polarization diversity combines pairs of antennas with orthogonal polarizations (i.e.horizontal/vertical, \pm slant $45^{\circ}$, Left-hand/Right-hand etc.). Reflected signals can undergo polarization changes depending on the medium through which they are travelling. A polarization difference of $90^{\circ}$ will result in an attenuation factor of up to $34 \mathrm{~dB}$ in signal strength. By pairing two complementary polarizations, this scheme can immunize a system from polarization mismatches that would otherwise cause signal fade [29].

\subsubsection{Frequency Diversity}

The signal is transmitted using several frequency channels or spread over a wide spectrum that is affected by frequencyselective fading [13]. The rationale behind this technique is that frequencies separated by more than the coherence bandwidth of the channel will not experience the same fade. The probability of simultaneous fade will be the product of the individual fading probabilities [34].

\subsection{Adaptive Optics (AO)}

As light from distant celestial objects enters our atmosphere it gets disturbed by our ever-moving atmosphere. Adaptive optics $(A O)$ corrects for the distortions in an image caused by this atmospheric turbulence. AO system operates at high frequencies, typically about $1000 \mathrm{~Hz}$. This is too fast for altering a primary mirror so adaptive optic systems are designed to act via the secondary mirror and additional optical elements placed in the light path [9].

\subsection{Beam Divergence}

Beam divergence purposely allows the beam to diverge or spread. The advantage of using narrow beam in FSO system generates much higher data rates and increases the security. Laser generated with extreme narrow light can be easily modulated with voice and data information. The beam spread is dependent on the beam divergence angle and transmission range. Typically, $1 \mathrm{mrad}$ to $8 \mathrm{mrad}$ beam divergence spreads 1 to $8 \mathrm{~m}$ at distance of $1 \mathrm{~km}$. To avoid spreading of a large beam, it is better to use narrow beam divergence such as 1mrad [11, 14, and 15].

\subsection{Bit Error Performance}

Bit error rate of the received signal is determined after propagation through cloud. It is observed from the transfer function of cloud that high attenuation of transmitted signal occurs while it passes through cloudy environment. Therefore there is higher probability of error due to inter symbol interference (ISI) which is occurred by pulse broadening in cloud. The inherent non -linear frequency response of cumulus cloud causing successive symbols to blur together. The presence of ISI in the system introduces errors in the decision device at the receiver output [16].

\subsection{Advanced DWDM FSO System}

In this scenario, various kinds of wireless signals can be transmitted by using DWDM full optical FSO links. A good transmission can be obtained by using DWDM. It is a technology that took data from different sources and put together on a channel, with each signal carried at the same time on its own separate light wavelength. Using DWDM, up to 96 wavelengths or channels of data can be multiplexed into a light stream which gives it to the laser beam for transmission $[27,28]$.

\section{FREQUENCY USED FOR FSO}

Frequency is a major concern for utilizing an FSO link. Two ranges are there that are 780-850nm and 1550-1600nm. The safety concern related to the effect of FSO beam to human eye and skin should be considered.

\section{0-850nm}

These wavelengths are suitable for FSO operation, and several vendors provide higher-power laser sources that operate in this region. At $780 \mathrm{~nm}$, inexpensive CD lasers are available, but the average lifespan of these lasers can be an issue and must be addressed during system design. At 850nm, reliable, inexpensive, high-performance transmitter and detector components are readily available like highly sensitive silicon (Si) avalanche photodiode (APD) detector technology and advanced vertical-cavity surface-emitting laser[18]. 


\section{0-1600nm}

These wavelengths are well suited for free-space transmission, and high quality transmitter and detector components are readily available. The combination of low attenuation and high component availability in this wavelength makes the development of wavelength-division multiplexing (WDM) FSO systems feasible. However, components are generally more expensive, and detectors are typically less sensitive and have a smaller receive surface area when compared with Si APD detectors that operate in the $850 \mathrm{~nm}$ wavelength [18].These wavelengths produce higher power and more eye safe compared to $780 \mathrm{~nm}$ and $850 \mathrm{~nm}$. Wavelength which is greater than $1400 \mathrm{~nm}$ is able to penetrate haze, smog, and fog [17]. In addition, these wavelengths are compatible with erbium-doped fiber amplifier (EDFA) technology, which is important for high-power $(>500 \mathrm{~mW})$ and high-data rate $(>2.5 \mathrm{Gbit} / \mathrm{s})$ systems. Finally, 50-65 times as much power can be transmitted at $1520-1600 \mathrm{~nm}$ than can be transmitted at 780-850nm for the same eye safety classification, owing to low transmission of the human eye at these wavelengths[11].

\section{MODULATION USED FOR FSO}

The choice of modulation technique for optical signal transmission plays an important role in reduction of turbulence. An important factor on the selection of modulation technique for FSO systems is the receiver sensitivity. Optical carrier can be frequency modulated, phase modulated or amplitude modulated.

The technically simplest digital modulation scheme is amplitude-shift keying (ASK). It does not offer immunity to the turbulence induced fading [19]. In optical systems it is referred to as on-off keying (OOK). OOK is an intensity modulation scheme where the light source (carrier) is turned on to transmit a logic "one" and turned off to transmit a "zero". In its simplest form this modulation scheme is called NRZ (non-return-to-zero)-OOK. Besides NRZ also other codes exist. The most common one besides NRZ is RZ (return to-zero) coding. The advantages of RZ compared to NRZ are its higher sensitivity and the fact that the clock frequency lies within the modulation spectrum [20].

The error probability expression over atmospheric channels is derived under the assumption of log-normal distributed scintillation, and then the performance of DPSK system is compared with that of a most commonly used on-off keying (OOK) system under the same channel conditions. Theory analysis and numerical results illustrate that with the same bandwidth, DPSK system has a higher sensitivity than OOK, and to a certain extent DPSK format can reduce the impairment from turbulence induced scintillation for its threshold being signal intensity insensitive, and hence, DPSK format is very suitable for atmosphere channels and has a broad prospect in wireless optical communications[21].

OOK RZ and OOK NRZ are mostly deployed techniquesdue to its efficient bandwidth usage, but others techniques can also be implemented. The external modulation gives better performance in comparison to direct modulation because direct NRZ spectrum has a strong carrier component compared to external modulated NRZ. RZ modulation format is best for long distance, but is complex and costly. Where NRZ is used for short distance and it is less complex, cheaper in comparison to $\mathrm{RZ}[2]$.

\section{CONCLUSION}

Free Space Optic offers solutions for current bottlenecks in communication technology. FSO have the potential to improve the standard of communication in a large number of applications due to benefits such as high bandwidth, high data rate, license free network, easy installation and many more. The effect of atmospheric scintillation on FSO link is an important issue and can be reduced byautomatic gain control mechanism. Performance of FSO link which is affected by turbulence can be enhanced by using diversity techniques, multiple transceivers, adaptive optics and many more. Choice of frequency is an important concern to set up FSO link, $850 \mathrm{~nm}$ or $1550 \mathrm{~nm}$ range can be utilized, $1550 \mathrm{~nm}$ is preferred due to human eye safety concern. OOK modulation scheme is mostly deploying for FSO link due to its efficient bandwidth usage but othermodulation likeDPSK, PSK can also be utilized.RZ modulation format is best for long distance,it is highly sensitive but is complex and costly. There is a great scope of further research in this field that will surely overcome a large number of issues faced in today's communication links.

\section{REFERENCES}

[1] A. Prokes, V. Skorpil, "Estimation of free space optics systems availability based on meteorological visibility", presented at IEEE Latin-American conference on Communications, 2009.

[2] Jitendra Singh, Naresh Kumar, "Performance analysis of different modulation format on free space optical communication system", 2013.

[3]I. I. Kim and E. Korevaar, "Availability of free space optics_FSO_and hybrid FSO/RF systems," Proc. SPIE 4530, 8495,2001 .

[4] http://www.ieee802.org/11;Jan 2007.

[5] Amarjeet Kaur, Ravinder Kumar Panchal, "Analysis the effect Atmosphere Turbulence in Free Space Optical (FSO) Communication Systems", International Journal of Engineering and Innovative Technology (IJEIT), Volume 3, Issue 11, May 2014.

[6] Ajaybeer kaur, Dr. M.L Singh, " Comparing the effect of Fog and Snow Induced attenuation on Free Space Optics(FSO) and RF Links", IJCST Vol. 3, 2012. 
[7]S. Bloom The Physics of Free Space Optics, AirFiber Inc.2 May 2002

[8] www.fsona.com

[9] http://www.atnf.csiro.au/outreach/education/senior/astrophysics/adaptive optics.html

[10] Heinz A. Willebrand, Baksheesh S. Ghuman, "fiber optics without fiber", IEEE Spectrum august 2001.

[11] Bloom, S., Korevaar, E., Schuster, J. and Willebrand, H ,Understanding the performance of free-space optics", Journal of Optical Networking, Optical Society of America, Vol. 2, Issue 6, 178-200, 2003.

[12] Arun K. Majumdar, Workshop on "Free-Space Laser Communications and Advanced FSO systems", GTU,Chandkheda Campus, Sept.,2013.

[13] wikipedia.org/wiki/Diversity_scheme

[14] I.I.Kim, B. McArthur and E. Korevaar, "Comparison of laser beam propagation at $785 \mathrm{~nm}$ and $1550 \mathrm{~nm}$ in fog and haze for optical wireless communications", Optical Wireless Communications III, SPIE, 4214, 2001.

[15] Jeganathan, Muthu and Pavellonov, "Multi-Gigabits per Second Optical Wireless Communications", 2000.

[16] Carlson A. B. (1986),“Communications System”, third edition, McGraw Hill, London, UK .Marshalek, R. G. and Koepf, G.A. (1988), "Optical technology for space communication system”, Opt. Eng .,pp 27, 663.

[17] B.S. Naimullah, M. Othman, A. K. Rahman, S.I.Sulaiman, S.Ishak, S.Hitam and S.A. Aljunid. "Comparison of Wavelength Propagation for Free Space Optical Communications". Research Gate, April 16,2014.

[18] http://web.mst.edu/ mobildat/Free\%20Space\%200ptics/

[19] T.T. Nguyen, L. Lampe, "Coded multi pulse pulse-position modulation for free-space optical communications", IEEE Trans.Commun, 58 (2010) 1036-1041.

[20] PAUER, M., WINTER, P., LEEB, W., "Bit error probability reduction in direct detection optical receivers using rz coding”, Journal of Light-wave Technology, 2001, vol.19, p. 1255-1262.

[21] Shalini Khare, Namrata Sahayam, " Analysis of Free Space Optical Communication System for Different Atmospheric Conditions \& Modulation Techniques”, International Journal of Modern Engineering Research (IJMER), Vol.2, Issue.6, pp4149-4152, Nov-Dec. 2012.

[22]http://www.engineersgarage.com/articles/wireless_communication

[23]Morgan Chen, "Review of Free-Space Optical Communications Links”, chenm@hkn.eecs.berkeley.edu, May 13th, 2003.

[24] Shruti R. et all. "Free Space Optics (FSO): Present Scenario andDevelopments", HCTL Open Science and Technology Letters (HCTL Open STL), Volume 4, February 2014, e-ISSN: 2321-6980 [25] Ghassemlooy Z. and Popoola W. O. (2010), "Terrestrial Free-Space Optical Communications", Mobile and Wireless Communications Network Layer and Circuit Level Design, Salma Ait Fares and Fumiyuki Adachi (Ed.), ISBN: 978-953307-042-1,

[26] Amandeep Kaur Virk et al., "Link Margin Optimization of Free SpaceOptical Link under the Impact of VaryingMeteorological Conditions", International Journal of Engineering Science and Technology (IJEST), Vol. 4 No.03 March 2012, ISSN: 0975-5462

[27] Tsukamoto, K.,S. Komaki, and M. Matsumoto, "Development project of radio on free space optics," Proc. SPIE, Jan. 2007.

[28] Mitsuji Matsumtoto: Next generation FSO system by system design optimization and performance enhancement; progress in electromagnetic research symposium proceedings, KL, Malaysia, march 2012.

[29] https://en.wikipedia.org/wiki/Antenna_diversity

[30] Abdulsalam Alkholidi, Khalil Altowij (2012), "Effect of Clear Atmospheric Turbulence on Quality of Free Space Optical Communications in Western Asia”, Optical Communications Systems, Dr. Narottam Das (Ed.), ISBN: 978-953-51-0170-3

[31] A. K. Majumdar, J. C. Ricklin, "Free Space Laser Communications Principles and Advances", Springer ISBN 978-0387-28652-5, 2008

[32] P. P. Smyth et. al., "Optical Wireless Local Area Networks Enabling Technologies", BT Technology Journal, 11(2), pp. 56-64, 1993.

[33] M. A. Bramson, "in Infrared Radiation", A handbook for Applications, Plenum Press, p. 602, 1969.

[34] http://www.srmuniv.ac.in 


\section{AUTHORS}

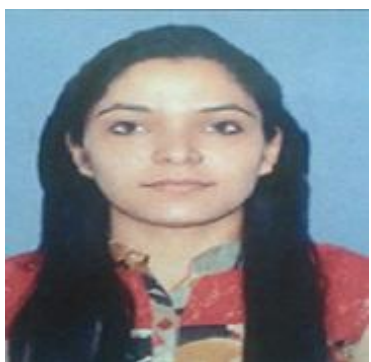

Er.Sushila Thakur was born in1991. She did her Bachelor's Degree in Electronics and Communication Engineering from SIRDA Institute of Engineering and Technology Sundernagar (Himachal Pradesh) in 2013 under HPU Shimla. She is pursuing her Master's degree in Electronics and Communication department from Eternal University, H.P, India. She is doing her M.Tech thesis under the guidance of Er. Rajeev Thakur. Her area of interest are Field of Optical Communications, Digital Electronics, Wireless Networks.

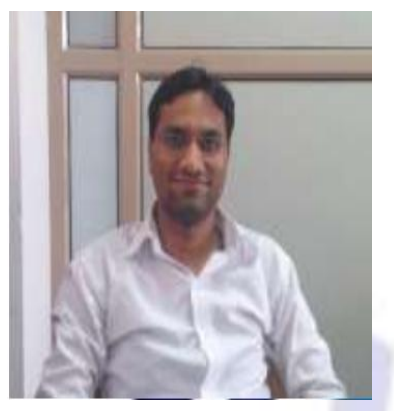

Er.Rajeev Thakur was born in1984. He received his Bachelor's Degree in Electronics and Communication Engineering from Institute of Engineering and Technology Baddi (Himachal Pradesh) in 2008 under HPU Shimla. He did his Master's degree in Electronics and Communication department from Eternal University, H.P, India. His area of interest are Field of Optical Communications, Digital Signal Processing, Wireless Networks and Embedded Systems.

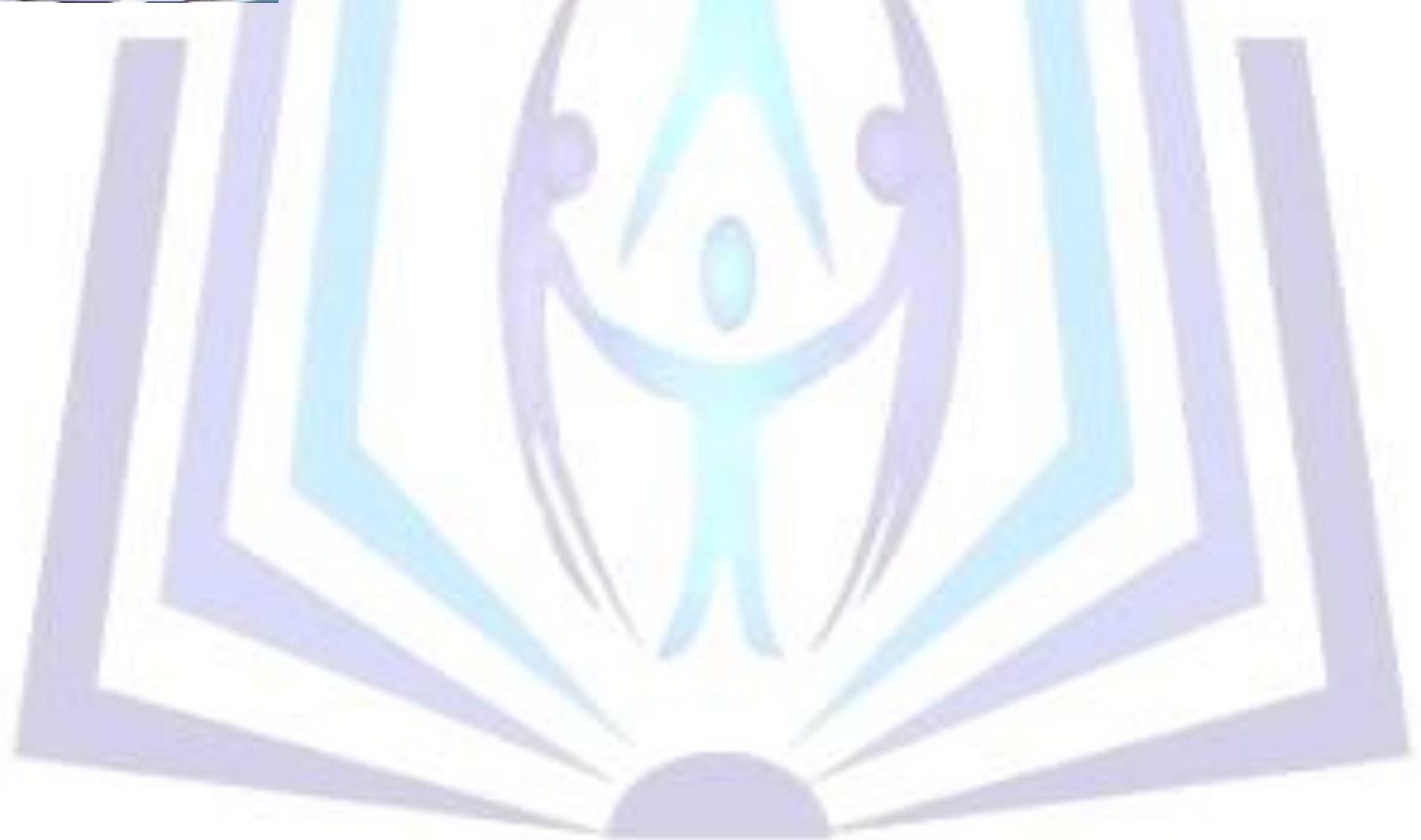

